\title{
A Framework for Hybrid Multimodal Performances
}

\author{
Shannon Cuykendall, Prophecy Sun, Reese Muntean, Thecla Schiphorst, Steve DiPaola \\ School of Interactive Arts + Technology, Simon Fraser University \\ 250-13450 102 Avenue Surrey, British Columbia V3T 0A3, Canada \\ scuykend@sfu.ca, pdsun@sfu.ca,rmuntean@sfu.ca, thecla@sfu.ca sdipaola@sfu.ca
}

\begin{abstract}
The boundaries between physical and virtual spaces are becoming more blurred in our everyday lives with the advent of wearable action cameras, virtual reality technologies, and algorithmicallyled audience interactions. Artists and creatives have been at the forefront of exploring these new technologies; however, little literature exists that reflects on best practices for navigating this new complex space. We reflect on our experiences in creating and participating in what we refer to as 'hybrid multimodal performances' or performances that blend physical and virtual spaces. We propose a framework of aesthetic choices we have implemented to seamlessly blend physical and virtual entities. We consider aspects such as the interaction between the camera and performer, the integration of multiple conceptual spaces, the changing relation between the artist and the work, and the multiple transformations of shape that occur when transitioning between physical and virtual spaces.
\end{abstract}

Dance. Video art. Virtual Reality. Bricolage. Decentralisation. Conceptual blending. Multiperspectivity.

\section{INTRODUCTION}

The development of aesthetic and production tools such as $360^{\circ}$ cameras, wearable action cameras, algorithmic processing techniques, and full virtual reality has opened new opportunities for artists to explore multiplicity - multiple roles, multiple transformations, multiple modalities, and multiple viewpoints. As this technology continues to improve, the options and space in which artists create in is becoming increasing more complex.

New camera technologies and virtual reality bring promise of providing a more immersive experience to audiences. Cho et al (2016) describe their process of creating a $360^{\circ}$ narrative dance film, Imago using 10 GoPro cameras. They describe how audiences experienced VR space differently than traditional film stating that audiences 'were unable to detach themselves from being part of the experience, as one might when watching a traditional film' (1).

To fully explore the capabilities of these new immersive technologies, we can no longer rely on traditional camera techniques, but must develop new practices and styles. We propose a framework for creating what we refer to as 'hybrid multimodal performances.' These performances include interactive and video-based work that seek to combine physical and virtual entities. We focus primarily on performances that include dance and movement as an integral component of the performance. Our framework includes four main areas of investigation. These will be further defined throughout the paper and include: performing with the camera, the aesthetic of bricolage, decentralisation, and the multiple transformations of shape that can arise when integrating physical and virtual spaces (see Table 1). We explore this framework in relation to previous hybrid multimodal performances we have participated in or created, and describe how this framework has led to the development of Fractured Perspectives: Movement and Light (FPML). This video artwork combines multiple perspectives of movement and dance from a $360^{\circ}$ camera, algorithmic video processing techniques, and multiple traditional cameras. The work will be presented at the British Computer Art Society in London, as a part of EVA 2017.

\section{PERFORMING WITH THE CAMERA}

With the advent of $360^{\circ}$ and wearable action cameras, artists have begun to explore not only new ways of performing for the camera, but also new capabilities of performing with the camera. The moving camera has been historically utilised to bring the audience closer to action in film. Brannigan (2011) states: 
Table 1: Framework for Multimodal Performances

\author{
Performing with the Camera \\ Videographer as Performer \\ Performer as Videographer
}

$\mid \begin{gathered}\text { Bricolage } \\ \text { Creating Constraints } \\ \text { Reconceptualisation }\end{gathered}$

The proximity of the camera to the action and its constant mobility certainly take the viewer close to what it feels like in such situations. The swooning, swilling actions of the performers and camera create a kinesthetic pattern...drawing the audience into a field of movement that has no firm anchor, replicating the floating, fuzzy sensations of intoxication (111).

Wearable or small handheld cameras provide for easy camera motion allowing for multiple viewpoints and perspectives to be captured. Reynolds (2013) describes her experience of watching the firstperson perspective of dancers in a performance:

\begin{abstract}
The effect of being immersed in a field of movement without visual stability and control is that I feel that vision becomes more 'porous' to other senses, particularly touch through contact with the floor in this scene, and also hearing, perhaps because of needing to draw on all available information in order to orientate oneself (223).
\end{abstract}

Reynolds' reflection suggests that the visual instability of the moving camera may help bring awareness to other senses and thus provide a more immersive experience for audiences.

Although new camera technologies may be able to provide more engaging experiences, many challenges remain in filming with these cameras. For example, when using a $360^{\circ}$ camera there is no "backstage" because there is no "behind the camera" and anything within view becomes part of the performance. Additionally, in $360^{\circ}$ video, the location of the camera becomes both the location of the audience and the centre of the scene, making it difficult to frame and emphasise important actions. Reynolds (2013) also reports on many negative feelings that a moving camera from the first-person perspective may elicit such as motion sickness or frustration from not being able to see the entire scene. Based on our experiences, we describe ways to overcome these challenges by more closely integrating videography into the performance.

\subsection{Performer as Videographer}

In Presence in a Box: Crossing Liminal Spaces (2016) the performer, Shannon Cuykendall wore a GoPro head-mounted camera while performing. The headcam in this performance was used to

Decentralisation
Abstracting Motion
Extending the Body

\begin{tabular}{|} 
Shape \\
Transformation \\
Unintentional Objects \\
Multiperspectivity
\end{tabular}

explore ways of communicating the feeling of presence to an audience. Cuykendall's first-person perspective video was played back to audiences during times that Cuykendall was not performing live. In the first-person view, the dancer's body is often out of frame and the movement of the camera and the spectators' reactions take centre stage. Cuykendall reflects:

I improvised the movement in the live performance, but was very aware of the camera. Therefore, I carefully planned the movement in relation to the camera. The camera became an extension of my body. I moved slowly and smoothly, and attempted not to make any sudden fast head movements. I emphasised moments of stillness and silence. My hands also became an important element, and I played with how they could frame the scene and come in and out of the camera's frame.

In this performance, Cuykendall developed a movement vocabulary specifically for the firstperson perspective to lessen negative emotions such as motion sickness that sometime accompany this perspective.

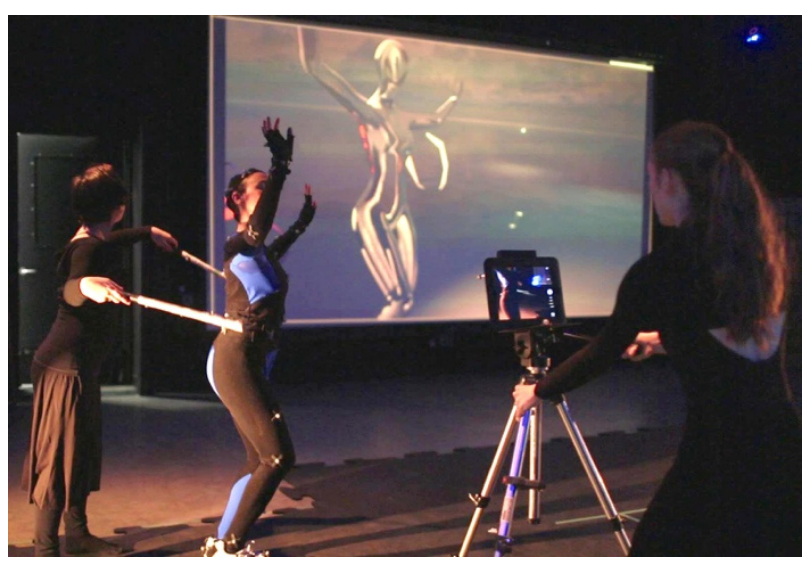

Figure 1: Performer as Videographer in Glass Dance (2015).

Cuykendall also took on a dual role as a performer and videographer in Glass Dance (2015). Cuykendall danced with a tablet placed on a wheeled tripod (see Figure 1). The tablet served as a viewfinder for a spatially aligned virtual camera. The perspective of the virtual camera was projected on a screen behind the performers. Movements of 
the camera in physical space translated to equivalent movements of the camera in the virtual world. Thecla Schiphorst created choreography for Cuykendall that consisted of specific movements as well as rules for how to frame the other performers in the virtual space. In contrast to Presence in a Box: Crossing Liminal Spaces (PIAB), the role of the camera in Glass Dance took on a role of another performer. Glass Dance and PIAB demonstrate two scenarios of how movement and the camera can become more integrated into the performance when the primary emphasis is on the choreography and not on documentation.

\subsection{Videographer as Performer}

The performer and camera can also be integrated in a performance when the main focus is on documentation. In worlds (2015), videographer Reese Muntean used the camera of a tablet to frame an aerial performer in a virtual world. The video that Muntean captured was displayed in real time on a large monitor behind the aerial performer. The monitor not only displayed what Muntean captured with the camera, but also placed the performer in a virtual space with other performers in remote locations.

In contrast to Cuykendall's role in Glass Dance, Muntean's movements were not intended as choreography and Muntean was instead tasked with filming and framing the view into the virtual world. While there were no direct interactions between Muntean and the aerial performer, she would approach the aerialist to explore certain "shots" of the virtual world. The performer was aware when Muntean was near and would position herself and her body towards the tablet. Muntean's status as a participant was further strengthened by this positioning between the audience and the aerialist. She moved about the space in front of the audience, sometimes even positioning herself on the far side of the aerialist.

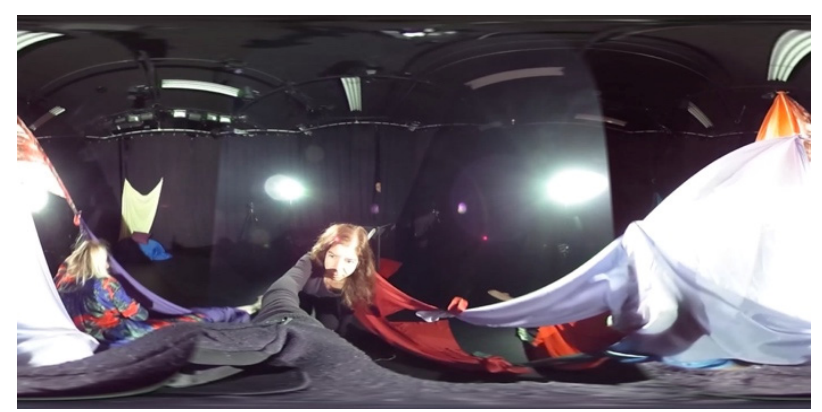

Figure 2: Videographer as Performer in Fractured Perspectives: Movement and Light (2017).

In FMPL, we continue to explore how the videographer can participate as a performer. We originally prioritised the role of the videographer for documentation, but through our iterations found that some performance was desirable and even necessary to have more control over how the $360^{\circ}$ camera framed the performers' movement. Steve DiPaola and Muntean took on the role of the videographer in FPML and wore black clothes and gloves while manipulating the $360^{\circ}$ camera (see Figure 2). As $360^{\circ}$ videographers in these improvisations, they attempted to blend in with the landscape and became accidental participants in the movement improvisation while navigating the space. Muntean notes:

\begin{abstract}
At first we tried to drape ourselves in the fabric and plastic sheeting to hide our faces and bodies as we took turns holding the $360^{\circ}$ camera in the space. With all of the movement of the dancers, I wanted to be able to travel around them as I might with a still or regular video camera. Once I started moving, through, the fabric fell away, and I would try to position myself somewhere in between the dancers. As they became entangled in the fabric and explored both the vertical space and ground level, I found myself similarly wrapped in the fabric and rolling on the floor to best capture a $360^{\circ}$ view of the improvisation.
\end{abstract}

In future filming sessions Muntean and DiPaola will be costumed in brightly coloured clothes and gloves to better coordinate with the dancers and props. In this way, rather than hiding the videographer or blending them into the background, they can blend into the performance itself.

\section{BRICOLAGE}

In hybrid, multimodal performance spaces the aesthetic of bricolage becomes a main area of investigation, as seemingly unconnected physical objects, movement, sound, and algorithmic processing techniques blend together to form new entities. Building from the definition put forth by anthropologist Claude Lévi-Strauss (1909-2009), the concept of bricolage is described as 'an in-situ practice of making with no plans but with an objective to create structure of what is happening through a material assembly' (Vallgårda \& Fernaeus 2015, pp. 175-176). Bricolage is often associated with postmodern art movements and emphasises aspects of daily life.

In artworks that combine the physical and virtual realms, the practice of bricolage takes on new meaning. Anything in physical space can be redefined and further transformed in the virtual realm. Objects and materials become transient, undefined entities that constantly change in meaning and form. Lévi-Strauss (1966) describes a bricoleur as one that creates 'by inclination or necessity' (13) within a set of constraints. However, in the virtual realm, many constraints cease to exist and artists are not limited to the physical objects that happen to be on hand or within reach. How do we create in a 
space with very few constraints and where anything that is conceivable is also possible?

In creating FPML we adopted a bricoleur attitude since we were experimenting with new technologies and techniques where the outcomes were often uncertain. Rather than beginning with a concept or plan of what the artwork was to be about, we instead created in a space where concepts were continuously redefined and emerged in-situ. This type of creation is challenging because it provides very little constraints to work within and base our artistic decisions. Therefore, early in the process we realised the need to define our own constraints.

\subsection{Creating Constraints}

\subsubsection{Spatial Constraints}

In FPML we chose to work in a black box studio with black curtains that could entirely encircle the performance space. This allowed us to create a "backstage" area when using the $360^{\circ}$ camera. We placed four video cameras on tripods around the space, framing the shots based on the location of three lights and the placement of objects in the space. With these framings, we could have multiple angles of the performers, and they could move through the performance area without concern for remaining in the frame of a particular camera.

We sculpted the physical space with physical objects and materials using traditional bricolage processes. We took objects that were on hand (e.g., garbage can, ballet barre, pillows, bean bag, table) and covered the objects with colourful fabric to create an abstract landscape. We hung fabric from the ceiling and used the fabric to physically connect dissimilar objects (see Figure 3). Additionally, we used two rolls of multi-purpose, medium-weight Poly Tarp plastic sheets. Each plastic sheet covered approximately 500 SQ.FT. We draped strips of the plastic onto multiple surfaces including the floor, various props, and objects.

The fabric, plastic, and objects provided occlusions in space that we had to navigate in, around, and between. In sculpting the physical space, we did not have a goal or objective to create a specific entity, but rather, we were informed by in-situ improvised quick decisions-making processes. Using these traditional methods of bricolage helped constrain many of our artistic choices.

\subsubsection{Sound Constraints}

In FPML Prophecy Sun created a pre-recorded sound score to accompany and inspire the experimental movements. The sounds blanketed the room and blended into the ever evolving physical and emotional landscape.
The sounds were looped and amplified through two small speakers placed behind the black curtains. The score consisted of three tracks: Moving time,

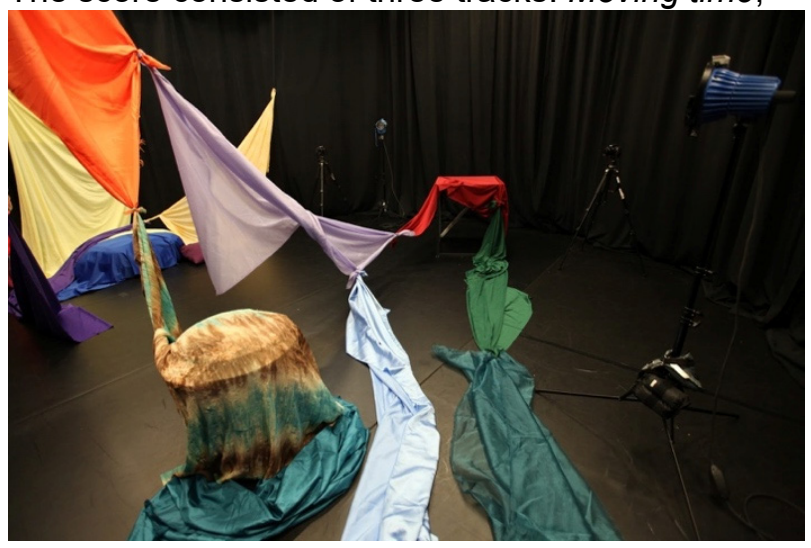

Figure 3: Spatial construction in FPML, photo by Reese Muntean.

Heartbeat, and Palace. Each track was recorded and mastered in post-production. Sun recorded the melodies with consumer technologies that included an iPhone, Behringer mixer, a pitch-shifting pedal, looping pedal and a high frequency microphone. Sun performed live in a studio. She layered improvisational vocal sounds over top of each other. The sounds were created using a variety of vocal and digital processing techniques such as throat singing, breathing in tandem sequences and fading in and out of soft pitch modulations. These pre-recorded looping sounds provided additional constraints in how we approached the work and helped set a consistent mood and tone among the performers.

\subsubsection{Movement Constraints}

We generated a movement score for FPML that was open enough to allow for exploration, but restrictive enough to allow for moments of congruency. Our movement was largely inspired by improvisation and Butoh techniques. Performers Cuykendall and Sun explored with notions of transformation (e.g., breathing, moving and falling into the back of the body), body scanning (e.g., firmly patting every part of the body in repetitive movements), and falling (e.g., controlled falling in spirals to the ground and upwards again).

The props we worked with also helped to structure movement tasks. For example, one task was to explore revealing and concealing ourselves within the plastic and fabric materials. Other tasks were defined loosely throughout the entire section, for example, we continuously explored repetition and mirroring one another.

\subsection{Reconceptualisation}

The act of integrating physical and virtual conceptual spaces to create a unified performance 
can be challenging. In creating FPML we embraced a constant state of reconceptualisation, as we transitioned between physical aspects of the performance and digital manipulations.

We used a Deep Dream Al painterly abstraction technique developed by DiPaola \& McCaig (2016). Using this technique, we can manipulate a video frame by changing the guide image, style layer, and the level of abstraction. Figure 4 demonstrates how the cloth-covered table in the lower left of the video frame becomes an insect-like creature, a tornado-like image, or a heart-like image depending on the guide image, style layer, and abstraction level that was applied. Varying the parameters allows us to adjust the bounds of the conceptual space we work in and each processed image develops new meaning to the work.

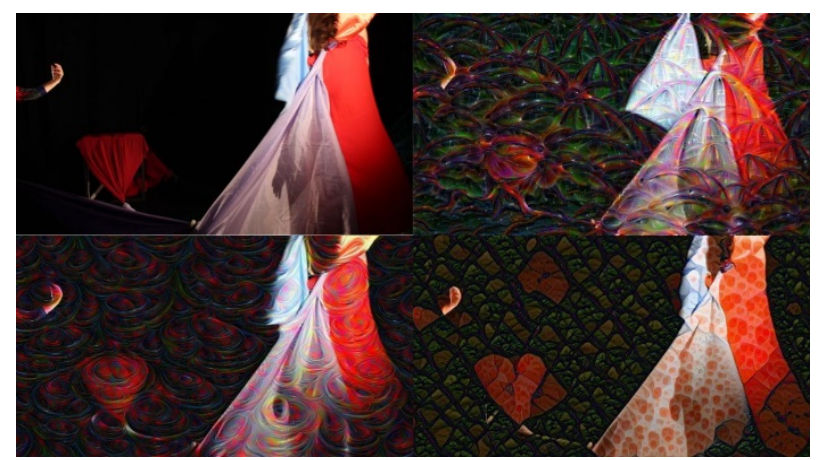

Figure 4: The cloth covered table in the top left image becomes multiple different shapes depending on the guide image, level of abstraction, and style layer.

Conceptual Blending is a proposed cognitive mechanism that allows for the creation of new concepts by integrating pre-existing conceptual spaces (Fauconnier \& Turner 1988). Al algorithms can be used to generate a synthesis of visuals by combining lower-level visual qualities such as images, shapes and textures with the higher-level concepts. The low-level qualities are extracted from the guide image and used to change the features of the source image. The layers of the network allow the production of blends with varying levels of intensity. With each layer of the network these lowlevel characteristics of the guide image can dominate the source image. Therefore, these $\mathrm{Al}$ abstraction systems allow us to conceptually blend on several fronts - from objects (e.g., dancers and props) that blend in and out of collaborative shapes over time and space to emergent use of colours, lighting and movement that blended via the abstraction process into a single whole.

In FPML we created a choreographic language based on how the $360^{\circ}$ camera and Al painterly abstraction techniques interpreted our movement. For example, we discovered that given the right conditions the Al would seamlessly blend parts of our bodies into the background (see Figure 5). With this in mind, we attempted to create movement that would cause conceptual blends in the Al software so that it would blur movement that emphasised the separation of our body and limbs to create specific effects (e.g., a hand that emerges out of nowhere). Our iterative process of transitioning between the physical and virtual realms to construct our movement vocabulary, allowed us to freely explore new techniques without having to adhere to any one conceptual idea.

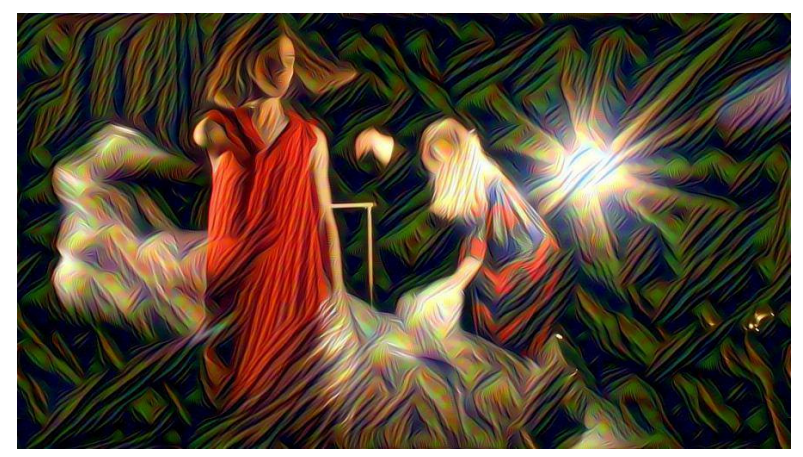

Figure 5: Video still from FPML (2017) demonstrating how performers blend into the environment.

\section{DECENTRALISATION}

In hybrid performance spaces control is distributed across many different entities. For example, in the case of FPML, the choreography of the movement is not just the movement of the body, but the movement of the camera, the interpretation of the movement by the $\mathrm{Al}$ algorithm, and other postprocessing video techniques. Therefore, the locus of movement shifts away from the body and becomes a collaboration between the body and machine.

This distribution of control leads to a vocabulary of movement that is separated from a concept of self or identity and is reminiscent of the concept of decentralisation, explored by Alwin Nikolais (1910-1993) in his modern dance technique (Nikolais \& Louis 2005, p. 9-12). Decentralisation describes a state where space, time, motion, and shape collide. Nikolais describes:

No longer dominated by personality and ego, the dancer now was able to merge into an environment of which he was a part. He found himself a contributing member rather than a dominating dweller (11).

In his choreography, Nikolais explored ways to abstract, conceal, or distort the human form and created "an overall visual composition" that did not privilege any one modality, such as, light, colour, pattern, or shape (Auslander 2005, p. 160). Auslander (2005) draws comparisons between Nikolais' technique and Clement Greenberg's 
(1909-1994) theories of abstract visual art. According to Greenberg, abstraction is a technique used by artists to direct the audience's attention to 'formal characteristics of the work' (158). Nikolais' techniques for abstracting the human body, therefore, allowed him to centre the audience's focus on the underlying motion of the work instead of the 'emotion' of the humans performing the work (Nikolais and Louis 2005, p. 10).

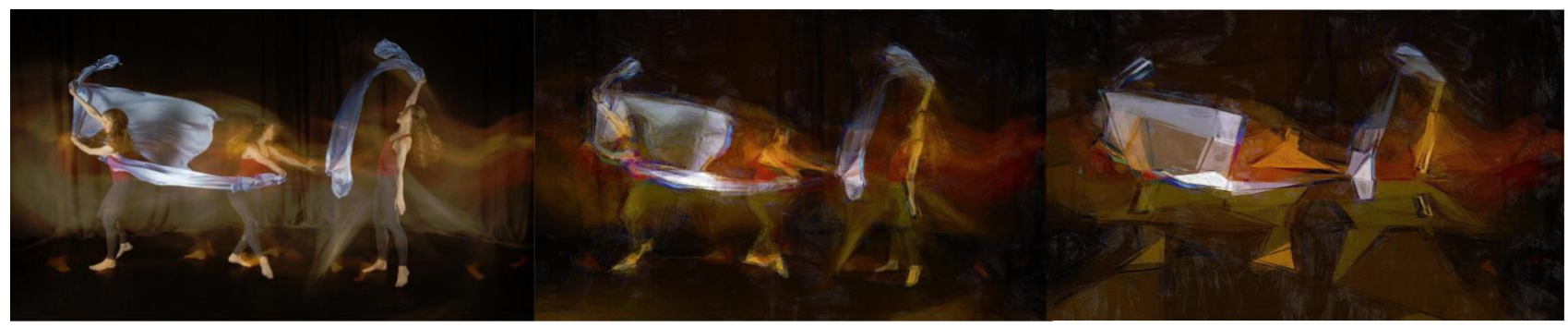

Figure 6: Cuykendall, DiPaola, and Muntean combined long exposure still photography with Al painterly abstraction.

Abstraction allows artists to uncover the fundamental components or essence of an object. Some artists have also suggested that what is often labelled as "abstract" is in fact a "more concrete version of our mental constructs' (Zimmer 2003, 1286). In a similar vein, Walter Benjamin (18921940) describes how cinema and photography can record aspects of reality outside of the range of normal human perception and becomes an avenue for experiencing the 'optical unconscious' (Benjamin 2000, p. 14-16).

We describe our use of video technology and algorithmic processing to aid in the abstraction process, thereby helping to uncover hidden truths and reality that lie just beyond the border of human comprehension. This method of working allows us to extend Nikolais' concept of decentralisation to not only mean a detachment of the ego from human movement, but also explore a new way of creating art that does not revolve around the human creator.

\subsection{Extending the Body}

Like Nikolais' work, we have explored ways in which the body's movement can blend into the environment. In PIAB Cuykendall and Sun left visible traces of their movement by attaching long strips of fabric to their costumes. In FPML we distorted our bodies into new non-human shapes while concealing ourselves in fabric and plastic. The malleable, lightweight texture of the plastic melded to our movements and often became static, clinging to our bodies, and acting as a second skin.

In FPML we also explored how we could connect our bodies to one another in $360^{\circ}$ virtual space. In a $360^{\circ}$ view the audience has a choice of where to look and performers on opposite sides of the room can appear distant and unconnected. Therefore, we used the fabric and plastic to connect our bodies across the entire space and form one unified structure.

\subsection{Abstracting Motion}

In addition to investigating how to abstract human bodies in the physical form, we have also explored with digital manipulation using $\mathrm{Al}$ painterly abstraction techniques. Cuykendall, Dipaola, and Muntean explored motion abstraction with the $\mathrm{Al}$ painterly technique in a 2015 dance photo shoot.

In this test shoot, strobe lights were incorporated into long exposure still photography to capture both key moments of a short, improvised dance sequence as well as the motion blur of Cuykendall moving through that sequence. This technique both merged the movements into a single visualisation a stream of colour that represented the totality of Cuykendall's dance through the space - and produced clear images of Cuykendall in various parts of the movement sequence (See Figure 6).

To achieve our final painterly style, we use our research into computational emotive painterly abstraction techniques including $\mathrm{Al}$ techniques of Genetic Programming and Deep Learning Neural Networks (DiPaola 2014a \& 2014b, DiPaola and McCaig 2016). The work uses Al techniques to learn cognitive creative art practice via analysis of historical art databases as well as encoding knowledge of art practice. While many Al art based systems of this type use a more template based approach, which limits the system to make one style or transfer style from a painting to a source, our system uses a more open perceptual based approach based on cognitive theories of human creativity (Salevati \& DiPaola 2015, Salevati et al. 2016).

Using Al painterly abstraction techniques in many ways frees the artist by allowing him or her to explore possibilities that can go beyond their human understanding. This also creates a shift in how the artist relates to the work in a similar manner to how Nikolais described the changing 
role of the dancer from a 'dominating dweller' to a 'contributing member.'

\section{SHAPE TRANSFORMATION}

In hybrid multimodal performances, new possibilities arise in relation to shape transformation. Camera technology inherently transforms the shape of a space depending on the field of view and lens one uses. In $360^{\circ}$ video, software aids in combining the perspectives from multiple individual cameras. This software manipulates the shape of the image to accommodate the desired view of the performance. Commonly provided views include rectilinear, fisheye, and a small planet options.

In addition to manipulating the shape of the image in a virtual realm, there are also considerations of how to integrate physical and virtual entities within the performance space. Artists can choose from a variety of projection surfaces and materials that all influence the overall shape of the performance. We focus this section on describing new techniques we have explored in transforming shape in the virtual realm.

\subsection{Unintentional Objects}

The Al painterly abstraction process we use in $F P M L$ allows us to experiment with different parameterisations of the concept of shape. Figures $4,5,6$, and 8 demonstrate how unintentional objects can be derived from an image using these abstraction techniques.

Shape is created by perception, and despite the apparent flexibility of shape in the painterly style, our Deep Learning Neural Network Al abstraction process has perceptually valid aspects of human creativity (DiPaola \& McCaig 2016). The Al painterly style makes explicit autonomous evaluations of the novelty and value of the source imagery as it abstracts. In FPML we worked to enhance these built in creative processes through our choices of lighting, colour, movement, and props.

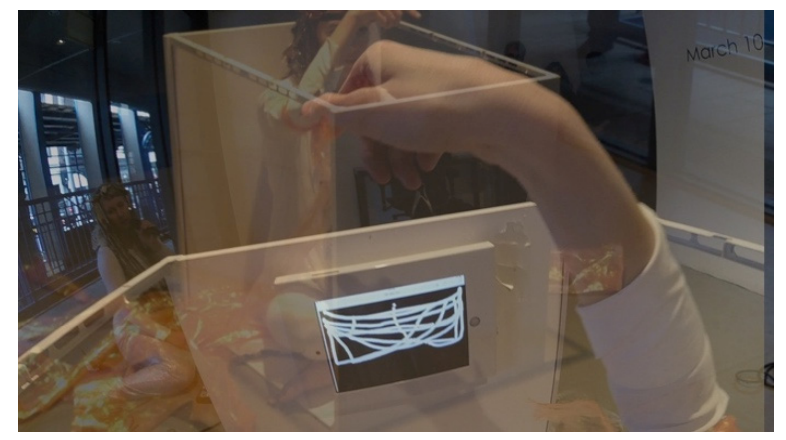

Figure 7: Video Still from PIAB Combining first and thirdperson perspectives.
How this Al creative process works can be viewed as important for creativity: for example, Jennings (2010) includes such autonomous evaluation as a necessary condition for creative autonomy. In terms of novelty, the abstraction system creatively isolates and maximizes subsets of the visual features evoked by the source video, resulting in the emergence and/or enhancement of certain aspect image qualities at the expense of other qualities. This process can be construed as the computation of one or more aesthetic value metrics. It bears a resemblance to neuroaesthetic principles of art, such as the notions of Zeki (2001) in highlighting/stimulating discrete portions of visual processing and translating the brain's abstractions on to the canvas. In this way, the abstraction system can bring out shapes and unintentional objects in ways associated with the human creative process.

\subsection{Multiperspectivity}

The notion of multiperspectivity, or presenting the audience with more than one perspective, was first explored in PIAB. In a video archive of the performance, Cuykendall combined third and firstperson perspectives of the performance (see Figure 7). In combining the first and third-person viewpoints, audiences are offered a unique perspective where one can both experience the movement of the dancer and see the entire work from a stable viewpoint.

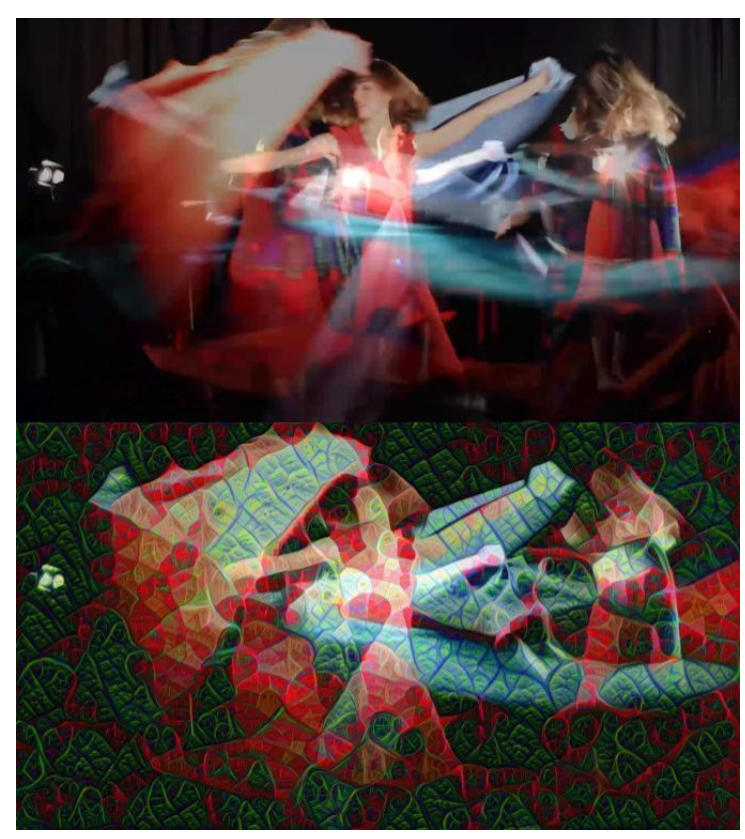

Figure 8: Shapes are further transformed when processing multiple perspectives with Al painterly abstraction techniques.

In FPML we captured movement from four traditional cameras and a $360^{\circ}$ camera, providing multiple different perspectives. We have combined the viewpoints captured with the traditional 
cameras in multiple ways including overlaying the viewpoints and splitting the frame to include each perspective side-by-side. These techniques, when combined with the Al painterly abstraction techniques, provide even more possibilities for shape transformation. Figure 8 demonstrates an Al painterly abstraction technique on a video frame consisting of multiple overlapping perspectives. The outcome of the process results in new shapes that combine our physical bodies, props, and multiple camera angles into one cohesive image.

\section{CONCLUSION}

Our framework considers the changing role of performers, creators, and technologies in hybrid multimodal performances. Based on anecdotal evidence and our previous experiences, we explore how the aesthetic of performing with the camera, bricolage, decentralisation, and shape transformation can come together to create a cohesive performance. We describe our process of transitioning between physical and virtual realms in FPML and reflect on how physical and virtual creative choices can not only inform one another throughout the creation process, but also blend seamlessly together to create new artistic practises, languages, and styles.

\section{REFERENCES}

Auslander, P. (2007) Motional Abstraction: Alwin Nikolais' Formalism. In Gitelman, C., \& Martin, R. (eds.), The Returns of Alwin Nikolais: Bodies, boundaries and the dance canon. Wesleyan University Press, Middletown, Connecticut.

Benjamin, W. (2008) The Work of Art in the Age of Mechanical Reproduction. Penguin UK.

Brannigan, E. (2011). Dance Film: Choreography and the Moving Image. Oxford University Press, New York.

Bowen, J. P., Diprose, G., and Lambert, N. (eds.) (2016) EVA London 2016: Electronic Visualisation and the Arts. Electronic Workshops in Computing, BCS. http://www.bcs.org/ewic/eva2016 (accessed 3 May 2017).

Carlson, K., Cuykendall, S., and Sun, P. (2016). Presence in a Box: Crossing Liminal Spaces. Scores + Traces: Exposing the Body through Computation. Exhibition, 10-12 March.

Cuykendall, S., DiPaola, S., Muntean, R., and Sun, P. (2017) Fractured Perspectives: Movement and Light. In Bowen, J. P., Diprose, G. \& Lambert, N. (eds.), EVA London 2017: Electronic Visualisation and the Arts. Electronic Workshops in Computing, BCS. (This volume.)

Cho, J., Lee, T. H., Ogden, J., Stewart, A., Tsai, T. Y., Chen, J., and Vituccio, R. (2016). Imago: presence and emotion in virtual reality. In ACM SIGGRAPH 2016 VR Village, Anaheim, California, USA, 24-28, article 6. ACM.
DiPaola, S. (2014a) Using a contextual focus model for an automatic creativity algorithm to generate art work, Procedia Computer Science, 41, pp. 212-219.

DiPaola S. (2014b) Computer modelling fine art painting using a cognitive correlative heuristics approach. In Proc: Biologically Inspired Cognitive Architectures. MIT, MA, USA.

DiPaola, S. and McCaig, G. (2016). Using artificial intelligence techniques to emulate the creativity of a portrait painter. In Bowen et al. (2016), pp. 158-165. DOI: 10.14236/ewic/EVA2016.32

Fauconnier, G., \& Turner, M. (1998). Conceptual integration networks. Cognitive Science, 22(2), 133187.

Goldberg, A. and Schiphorst, T. (2015). Glass Dance. Vancouver, BC, Canada.

http://www.peppersghost.org/?page id=45 (accessed 3 May 2017).

Goldberg, A., Schiphorst, T., Lantin, M., and Landry, T. (2015). Worlds. Vancouver, BC, Canada. http://www.peppersghost.org/?page id=43 (accessed 3 May 2017).

Jennings, K. E. (2010) Developing creativity: Artificial barriers in artificial intelligence. Minds and Machines, 20(4), pp. 489-501.

Lévi-Strauss, C. (1966) The Savage Mind. Chicago, IL: University of Chicago Press.

Nikolais, A. and Louis, M. (2005) The Nikolais/Louis Dance Technique: A Philosophy and Method of Modern Dance. Routledge, New York.

Reynolds, D. (2013) Empathy, contagion, and affect: The role of kinesthesia in watching dance. In Brandstetter, G., Egert, G. \& Zubarik, S. (eds.), Touching and being touched: Kinesthesia and empathy in dance and movement. Walter de Gruyter, Berlin/Boston.

Salevati S. and DiPaola S, (2015) A creative artificial intelligence system to investigate user experience, affect, emotion and creativity. In Ng, K., Bowen, J. P. \& Lambert, N. (eds.), EVA London 2015: Electronic Visualisation and the Arts, pp. 140-152. Electronic Workshops in Computing, BCS. DOI: 10.14236/ewic/eva2015.13

Salevati, S., DiPaola, S., Carlson, K., and Schiphorst, T. (2016) Movement awareness through emotion based aesthetic visualisation. In Bowen et al. (2016), pp. 124-132. DOI: $10.14236 /$ ewic/EVA2016.27

Vallgårda, A. and Fernaeus, Y. (2015). Interaction design as a bricolage practice. In Proceedings of the Ninth International Conference on Tangible, Embedded, and Embodied Interaction, January, pp. 173-180. ACM.

Zeki, S. (2001) Artistic creativity and the brain. Science, 293(5527), pp. 51-52.

Zimmer, R. (2003). Abstraction in art with implications for perception. Philosophical Transactions of the Royal Society of London B: Biological Sciences, 358(1435), pp. 1285-1291. 